\title{
Disseminated Herpes Simplex Virus Type 2 after Treatment with Idelalisib
}

\author{
Joseph Attallah*, Uqba Khan, Mamta Sharma and Zyad Kafri \\ Departments of Internal Medicine, USA
}

*Corresponding author: Joseph Attallah, Departments of Internal Medicine, Infectious disease and Hematology/Oncology St. John

Hospital and Medical Center, USA

\begin{tabular}{l}
\hline ARTICLE INFO \\
\hline Received: 㗀 January 30, 2019 \\
Published: 幽 February 13, 2019
\end{tabular}

Citation: Joseph Attallah, Uqba Khan, Mamta Sharma, Zyad Kafri. Disseminated Herpes Simplex Virus Type 2 after Treatment with Idelalisib. Biomed J Sci \& Tech Res 14(3)-2019. BJSTR. MS.ID.002560.

Abbreviations: CLL: Chronic Lymphocytic Leukemia; HSV: Herpes Simplex Virus; DLBCL: Diffuse Large B-Cell Lymphoma; FCR: Fludarabine, Cyclophosphamide and Rituximab

\section{ABSTRACT}

Introduction: Case reports describing disseminated Herpes simplex virus type 2 (HSV-2) disease are limited. To our knowledge, this is the first report of disseminated HSV-2 infection in a patient who was treated by idelalisib.

Case: A 62-year-old female with chronic lymphocytic leukemia (CLL) presented with a painful pruritic rash. Her skin exam showed multiple erythematous papular lesions with central necrosis. Polymerase chain reaction in the peripheral blood and skin lesions was positive for HSV-2. The diagnosis of idelalisib-associated disseminated HSV-2 was made and the patient was started on IV acyclovir. On follow-up, her skin lesions completely resolved in six weeks. Idelalisib was stopped and the patient was started on venetoclax.

Discussion: Idelalisib is a highly specific phosphatidylinositol 3-kinase delta (PI3K $\delta$ ) inhibitor that was approved by the FDA for the treatment of relapsed chronic lymphocytic leukemia, in combination with rituximab, relapsed follicular B-cell nonHodgkin lymphoma, and in relapsed small lymphocytic lymphoma. This case highlights the potential cutaneous manifestation in immunocompromised individuals, which is often atypical and poses a difficulty in diagnosis. Physicians should consider HSV-2 as a rare but important cause of a disseminated rash in immunocompromised individuals. Antivirals including acyclovir and valacyclovir can successfully treat HSV-2 associated skin infection.

\section{Introduction}

Case reports describing disseminated Herpes simplex virus type 2 (HSV-2) disease are limited. We present a case of disseminated (HSV-2) cutaneous disease in a 62-year- old female with a history of diffuse large B-cell lymphoma who began idelalisib three weeks prior to her presentation. To our knowledge, this is the first report of disseminated HSV-2 infection in a patient who was treated by idelalisib.

\section{Case Report}

A 62-year-old female with a history of chronic lymphocytic leukemia (CLL) with Richter's transformation to diffuse large B-cell lymphoma presented with a painful pruritic rash on her extremities, low-grade fever, fatigue and loss of appetite. The patient was diagnosed with stage 1 chronic lymphocytic leukemia in 2006. She had six cycles of fludarabine, cyclophosphamide, and rituximab
(FCR) and achieved a complete remission. In 2012 she developed a left inguinal lymph node, and a biopsy showed progression to diffuse large B-cell lymphoma (DLBCL). The patient received six cycles of rituximab, cyclophosphamide, doxorubicin, vincristine, prednisone, and rituximab (R-CHOP) treatment. A PET scan showed a new uptake in neck, axilla and subcutaneous right lateral chest wall. Two cycles of platinum-based salvage chemotherapy with rituximab, ifosfamide, carboplatin, and etoposide (RICE) were given. Follow-up PET scan showed progression of the disease so two cycles of cyclophosphamide, vincristine, doxorubicin, and dexamethasone (Hyper-CVAD) plus rituximab were given. The patient had progression of her disease in the left cervical area, so salvage therapy with rituximab and ibrutinib was started. Subsequently she developed pancytopenia, so ibrutinib was switched to oral idelalisib $150 \mathrm{mg}$ twice daily. Three weeks after initiating idelalisib she presented 
with painful pruritic rash. She was tachycardic and febrile, with a temperature of $102.20 \mathrm{~F}$.

Her skin exam showed multiple erythematous papular lesions with central necrosis on all extremities and on her back (Figures 1 \& 2). There were no vesicles, blisters or pustules identified on examination. CBC revealed hemoglobin of $10.3 \mathrm{~g} / \mathrm{dL}, \mathrm{MCV} 95.4 \mathrm{fL}$, platelet count $18,000 / \mu \mathrm{L}$, white blood cell count $3,400 / \mu \mathrm{L}$, and absolute neutrophil count 140/ $\mu \mathrm{L}$. IgG count was $706 \mathrm{mg} / \mathrm{dL}$, and her metabolic panel and liver enzymes were normal. Differential diagnosis included acute febrile neutrophilic dermatosis drug infection, drug eruptions, allergic reaction, and cutaneous lymphoma Workup included bacterial and fungal culture, polymerase chain reaction (PCR) for Epstein-Barr virus, cytomegalovirus, and herpes simplex virus. Polymerase chain reaction in the peripheral blood and skin lesions was positive for HSV-2. The diagnosis of idelalisib-associated disseminated HSV-2 was made and the patient was started on IV acyclovir. On follow-up, her skin lesions completely resolved in six weeks. Idelalisib was stopped and the patient was started on venetoclax for treatment of CLL.

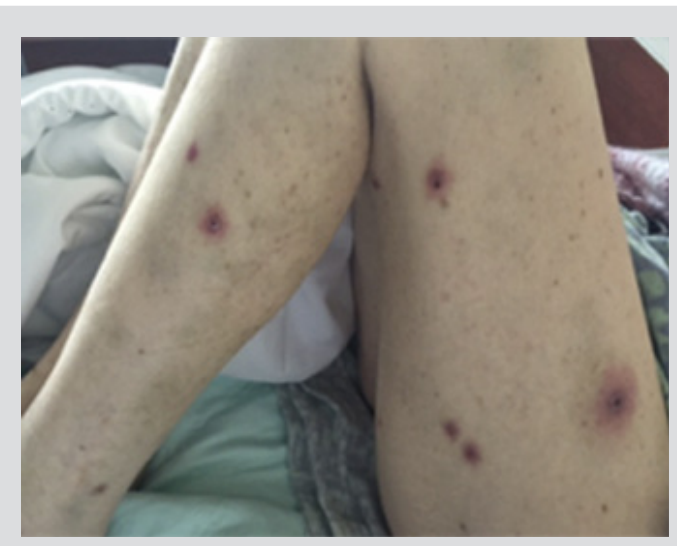

Figure 1: Multiple erythematous papular lesions.

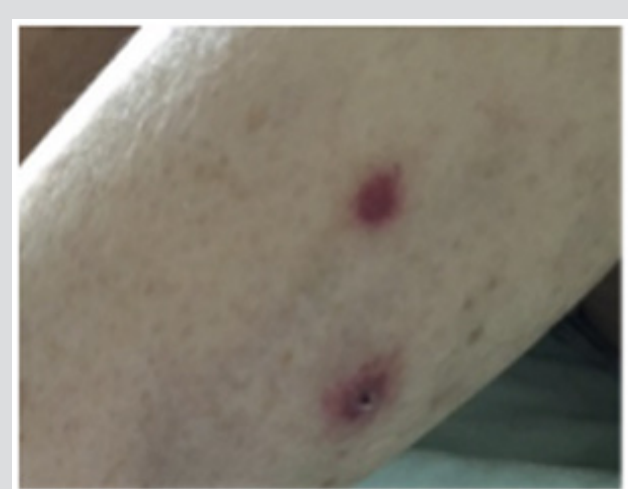

Figure 2: Central necrosis.

\section{Discussion}

Idelalisib is a highly specific phosphatidylinositol 3-kinase delta (PI3K $\delta$ ) inhibitor $[1,2]$ that was approved by the FDA in 2014 for the treatment of relapsed chronic lymphocytic leukemia, in combination with rituximab, relapsed follicular B-cell non-Hodgkin lymphoma, and in relapsed small lymphocytic lymphoma [3]. Prescribing information recommends monitoring patients for the development of severe cutaneous reactions and to discontinue if they occur. Cutaneous side effects that were reported in idelalisib-treated patients include dermatitis exfoliative, maculopapular rash, exfoliative rash, and toxic epidermal necrosis [4]. Cutaneous HSV-2 has never been previously reported. Other side effects that were associated with idelalisib are hepatotoxicity, severe colitis, intestinal perforation, and pneumonitis [4]. In 2016, FDA Alerts Health Care Professionals included reports of an increased rate of adverse events including deaths in clinical trials with idelalisib in combination with other cancer medicines. Six clinical trials were stopped as a result of these adverse reactions. This case highlights the potential cutaneous manifestation in immunocompromised individuals. The cutaneous presentation in these patients is often atypical and poses a difficulty in diagnosis. Physicians should consider HSV-2 as a rare but important cause of a disseminated rash in immunocompromised individuals. Antivirals including acyclovir and valacyclovir can successfully treat HSV-2 associated skin infection with complete resolution of skin lesions.

\section{References}

1. Lannutti BJ, Meadows SA, Herman SE, Kashishian A, Steiner B, et al. (2011) CAL-101, a p110delta selective phosphatidylinositol-3-kinase inhibitor for the treatment of B-cell malignancies, inhibits PI3K signaling and cellular viability. Blood 117(2): 591-594.

2. Durand CA, Hartvigsen K, Fogelstrand L, Shin Kim, Sally Iritani, et al. (2009) Phosphoinositide 3-kinase p110 $\delta$ regulates natural antibody production, marginal zone and B-1 B cell function, and autoantibody responses. J Immunol 183(9): 5673-5684.

3. (2014) US Food and Drug Administration. FDA approves Zydelig for three types of blood cancers. Press release.

4. ZYDELIG (2014) (idelalisib tablets). Full prescribing information, Gilead Sciences, Inc, Foster City, CA, USA. 


\section{ISSN: 2574-1241}

DOI: 10.26717.BJSTR.2019.14.002560

Joseph Attallah. Biomed J Sci \& Tech Res

(C) (i) This work is licensed under Creative

Submission Link: https://biomedres.us/submit-manuscript.php

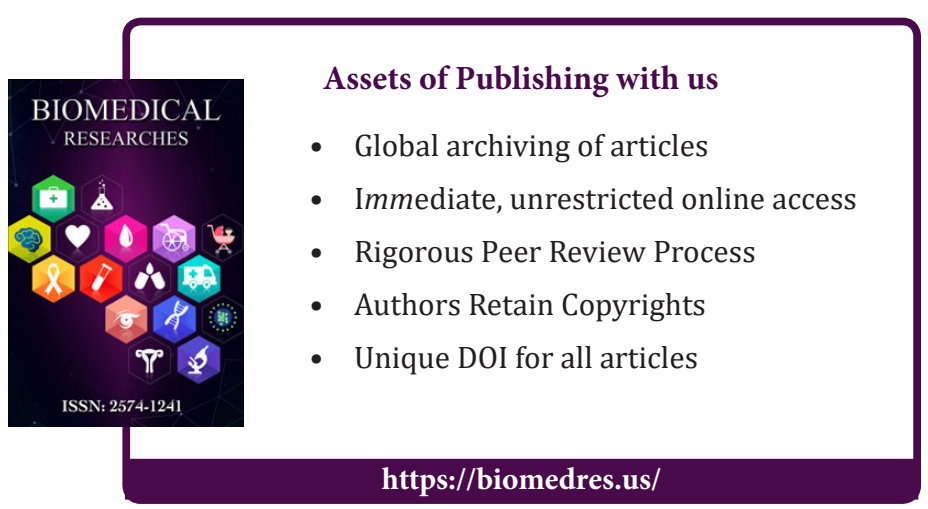

